\title{
Some Estimates on the Boltzmann Collision Operator
}

\author{
Jens Struckmeier \\ Department of Mathematics \\ University of Kaiserslautern \\ Germany
}

\begin{abstract}
The paper presents some new estimates on the gain term of the Boltzmann collision operator. For Maxwellian molecules, it is shown that the $L^{\infty}$-norm of the gain term can be bounded in terms of the $L^{1}$ and $L^{\infty}$-norm of the density function $f$. In the case of more general collision kernels, like the hard-sphere interaction potential, the gain term is estimated pointwise by the $L^{\infty}$-norm of the density function and the loss term of the Boltzmann collision operator.
\end{abstract}

\section{Introduction}

The Boltzmann equation for monoatomic gases given in the form

$$
f_{t}+v \nabla_{x} f=Q(f)
$$

is a nonlinear transport equation which describes the time evolution of a rarefied gas. In (1.1), the collision operator $Q(f)$ can be expressed in the form

$$
Q(f)=Q_{+}(f)-f L(f)
$$

with

$$
\begin{aligned}
L(f) & =\int_{\mathbb{R}^{3}} \int_{S_{+}^{2}} k\left(\left\|v-v_{*}\right\|, n\right) f\left(v_{*}\right) \mathrm{d} n \mathrm{~d} v_{*}, \\
Q_{+}(f) & =\int_{\mathbb{R}^{3}} \int_{S_{+}^{2}} k\left(\left\|v-v_{*}\right\|, n\right) f\left(v^{\prime}\right) f\left(v_{*}^{\prime}\right) \mathrm{d} n \mathrm{~d} v_{*}
\end{aligned}
$$

Here, $k\left(\left\|v-v_{*}\right\|, n\right)$ denotes some appropiate collision kernel and the postcollisional velocities are given by the collision transformation (written explicitely in the next section). The models for the collision kernel used in the following are of the form

$$
k\left(\left\|v-v_{*}\right\|, n\right)=\left\|v-v_{*}\right\| \cos \theta_{1},
$$


where $\left(v-v_{*}, n\right)=\cos \theta_{1}$.

In the following two sections we derive two new estimates for the gain term $Q_{+}(f)$, which are obtained without introducing any kind of truncation. In Section 2 we consider the case of Maxwellian molecules and prove the estimate

$$
\left\|Q_{+}(f)\right\|_{\infty} \leq C\|f\|_{\infty}\|f\|_{1},
$$

which is based on an appropiate transformation of $Q_{+}$in the center of mass system. The more general form of the collision kernel as given in (1.2) is discussed in Section 3. Here, it is obvious that $Q_{+}$is - in general - unbounded in $L^{\infty}$ (see Remark 3.4) and we prove a pointwise estimate of $Q_{+}$in the form

$$
Q_{+}(f)(v) \leq C\|f\|_{\infty} L(f)(v) \quad \forall v \in \mathbb{R}^{3} .
$$

\section{Estimate for Maxwellian Molecules}

In this section we are concerned with the gain term $Q_{+}(f)$ of the Boltzmann collision operator for Maxwellian molecules given in the form

$$
Q_{+}(f)=4 \int_{\mathbb{R}^{3}[0, \pi / 2]} \int_{[0,2 \pi]} f\left(v^{\prime}\right) f\left(v_{*}^{\prime}\right) \cos \theta_{1} \mathrm{~d} \phi_{1} \sin \theta_{1} \mathrm{~d} \theta_{1} \mathrm{~d} v_{*},
$$

where

$$
\begin{aligned}
v^{\prime} & =v-\left(v-v_{*}, n_{1}\right) n_{1} \\
v_{*}^{\prime} & =v_{*}+\left(v-v_{*}, n_{1}\right) n_{1}
\end{aligned}
$$

and $\left(v-v_{*}, n_{1}\right)=\cos \theta_{1}$. For the following it is more appropiate to consider the operator $Q_{+}$in the center of mass system, i.e. instead of the collision transformation (2.4), (2.5), we consider the form

$$
\begin{aligned}
v^{\prime} & =\frac{v+v_{*}}{2}-\frac{\left\|v-v_{*}\right\|}{2} n \\
v_{*}^{\prime} & =\frac{v+v_{*}}{2}+\frac{\left\|v-v_{*}\right\|}{2} n
\end{aligned}
$$

where $n \in S^{2}$ with polar angles $(\theta, \phi)$ may be obtained substituting $\theta=2 \theta_{1}$. Then, the operator $Q_{+}(f)$ can be expressed in the form

$$
Q_{+}(f)=\iint_{\mathbb{R}^{3}} \int_{[0, \pi]} \int_{[0,2 \pi]} f\left(v^{\prime}\right) f\left(v_{*}^{\prime}\right) \mathrm{d} \phi \sin \theta \mathrm{d} \theta \mathrm{d} v_{*}
$$

Applying the transformation $w=\left(v-v_{*}\right) / 2$, equation (2.6) can be written in the form

$$
Q_{+}(f)=2^{3} \int_{\boldsymbol{R}^{3}} \int_{S^{2}} f(v-w-\|w\| n) f(v-w+\|w\| n) \mathrm{d} n \mathrm{~d} w
$$


and passing to spherical coordinates for the vector $w$ in (2.7) leads to

$$
Q_{+}(f)=2^{3} \iint_{\boldsymbol{R}_{+}} \int_{S^{2}} z_{S^{2}} z^{2} f\left(v-z\left(n_{1}+n_{2}\right)\right) f\left(v-z\left(n_{1}-n_{2}\right)\right) \mathrm{d} n_{1} \mathrm{~d} n_{2} \mathrm{~d} z,
$$

where $n_{1}$ and $n_{2}$ are two unit vectors on $S^{2}$. Simple geometric considerations show, that the set $\left\{n_{1}+n_{2}: n_{1}, n_{2} \in S^{2}\right\}$ is a ball around the origin with radius 2 , i.e.

$$
\left\{n_{1}+n_{2}: n_{1}, n_{2} \in S^{2}\right\}=B_{2}(0)=\left\{x \in \mathbb{R}^{3}:\|x\| \leq 2\right\}
$$

and for fixed $x \in B_{2}(0)$,

$$
\begin{aligned}
& \left\{n_{1}-n_{2}: n_{1}, n_{2} \in S^{2}, n_{1}+n_{2}=x\right\}=S_{x}^{1} \\
& \quad=\left\{y \in I R^{3}:(y, x)=0,\|y\|=\left(1-\left(\frac{\|x\|}{2}\right)^{2}\right)^{1 / 2}\right\}
\end{aligned}
$$

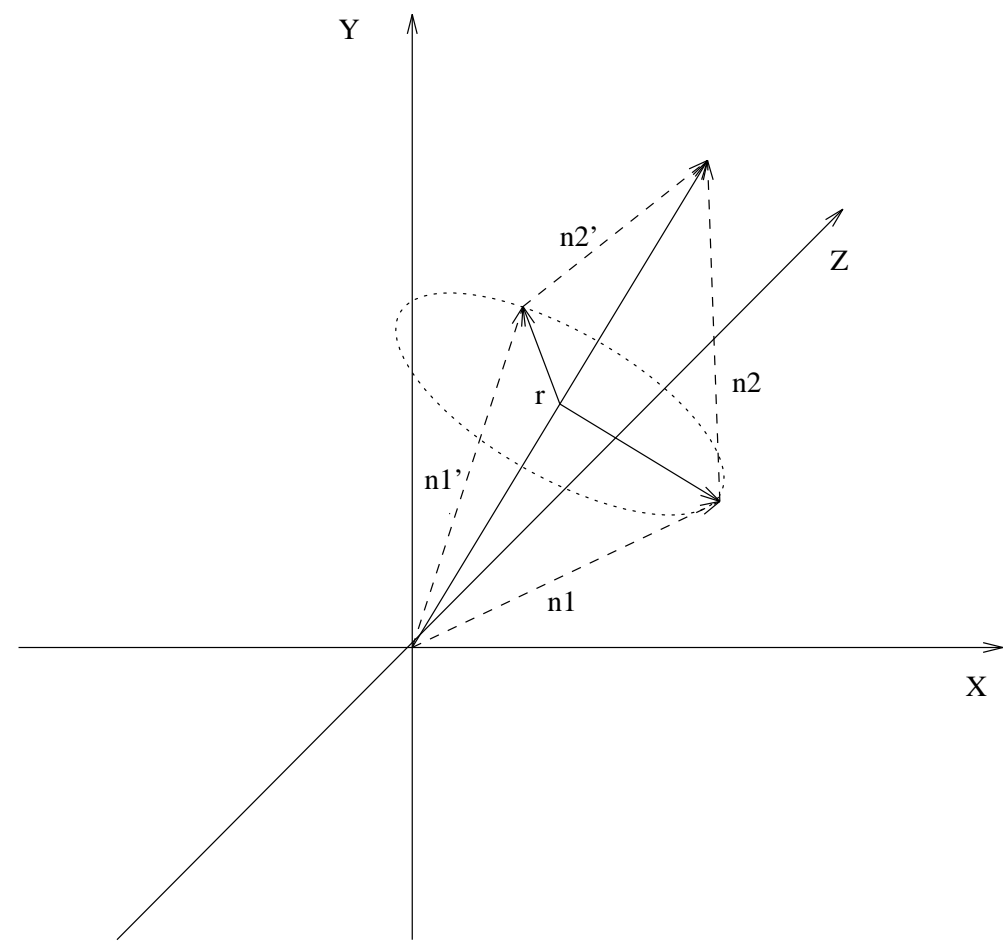

Hence, we have a transformation $T: S^{2} \times S^{2} \rightarrow\left\{(x, \xi): x \in B_{2}(0), \xi \in[0,2 \pi)\right\}$, where $\xi$ acts as a parametrization of $S_{x}^{1}$. Using the transformation $T$ we will show in the following that the gainterm $Q^{+}$can be written in the form

$$
Q_{+}(f)=2^{3} \int_{\mathbb{R}_{+}} \int_{[0,2]} \int_{S^{2}} \int_{S_{x}^{1}} z^{2} f(v-z r n) f(v-z a D(n) u) \mathrm{d} \xi \mathrm{d} \phi \sin \theta \mathrm{d} \theta r \mathrm{~d} r \mathrm{~d} z,
$$


where $n=(\sin \theta \cos \phi, \sin \theta \sin \phi, \cos \theta), D(n)$ a rotation matrix given by

$$
D(n)=\left(\begin{array}{ccc}
\cos \theta \cos \phi & -\sin \phi & \sin \theta \cos \phi \\
\cos \theta \sin \phi & \cos \phi & \sin \theta \sin \phi \\
-\sin \theta & 0 & \cos \theta
\end{array}\right)
$$

and

$$
\begin{aligned}
& u=(\cos \xi, \sin \xi, 0), \quad \xi \in[0,2 \pi) \\
& a=\left(4-r^{2}\right)^{1 / 2}
\end{aligned}
$$

Remark 2.1

Unless otherwise quoted, we assume in the following, that a unit vector $n \in S^{2}$ is expressed in the spherical coordinates

$$
n=(\sin \theta \cos \phi, \sin \theta \sin \phi, \cos \theta),
$$

where $\theta \in[0, \pi]$ and $\phi \in[0,2 \pi]$.

Using spherical coordinates for $x \in B_{2}(0)$, we found the following identities between $\left(n_{1}, n_{2}\right)$ and $(x, \xi)$.

\section{Proposition 2.2}

The transformation $T^{-1}$ is given by

$$
\begin{aligned}
& n_{1}=\frac{x}{2}+\frac{a}{2} D(n) u \\
& n_{2}=\frac{x}{2}-\frac{a}{2} D(n) u
\end{aligned}
$$

where

$$
x=r \cdot n, \quad n \in S^{2}
$$

$u$ defined by (2.9), a by (2.10) and the rotation matrix $D(n)$ by (2.8).

\section{Proof}

Consider the local coordinate system, such that $x=(0,0, r)$. Then, for $n_{1}+n_{2}=x$, we have

$$
\begin{aligned}
& n_{1}=\frac{1}{2}(a \cos \xi, a \sin \xi, r) \\
& n_{2}=\frac{1}{2}(-a \cos \xi,-a \sin \xi, r),
\end{aligned}
$$

where $\xi \in[0,2 \pi)$. Equations (2.11), (2.12) follow from passing to a global coordinate system via the rotation matrix $D(n)$ as given in (2.8).

\section{Proposition 2.3}

$$
\left|\operatorname{det} J T^{-1}\right|=r \sin \theta
$$




\section{Proof}

By Proposition 2.2, the unit vectors $n_{1}, n_{2}$ can be written in the form

$$
\begin{aligned}
& n_{1}=\frac{1}{2}(r \sin \theta \cos \phi+X, r \sin \theta \sin \phi+Y, r \cos \theta+Z) \\
& n_{2}=\frac{1}{2}(r \sin \theta \cos \phi-X, r \sin \theta \sin \phi-Y, r \cos \theta-Z)
\end{aligned}
$$

where

$$
\begin{aligned}
X(r, \theta, \phi, \xi) & =a \cos \theta \cos \phi \cos \xi-a \sin \phi \sin \xi \\
Y(r, \theta, \phi, \xi) & =a \cos \theta \sin \phi \cos \xi+a \cos \phi \sin \xi \\
Z(r, \theta, \xi) & =-a \sin \theta \cos \xi
\end{aligned}
$$

To compute $\left|\operatorname{det} J T^{-1}\right|$, we consider two sucessive transformations $T_{1}$ and $T_{2}$ defined as follows: We introduce the transformation $T_{1}:[-1,1]^{4} \rightarrow[0, \pi]^{2} \times[0,2 \pi]^{2}$ given by

$$
\begin{aligned}
& \theta_{1}=\arccos z_{1} \\
& \theta_{2}=\arccos z_{2} \\
& \phi_{1}=\arccos \left(\frac{x_{1}}{\sqrt{1-z_{1}^{2}}}\right) \\
& \phi_{2}=\arccos \left(\frac{x_{1}}{\sqrt{1-z_{2}^{2}}}\right)
\end{aligned}
$$

Straightforward computations yield

$$
\operatorname{det} J T_{1}=\frac{1}{\left|y_{1} y_{2}\right|}
$$

where $y_{1}\left(y_{2}\right)$ is the second component of $n_{1}\left(n_{2}\right)$. Now, the transformation $T_{2}$ is given by

$$
\begin{aligned}
x_{1} & =\frac{1}{2}(r \sin \theta \cos \phi+X(r, \theta, \xi, \phi)) \\
x_{2} & =\frac{1}{2}(r \sin \theta \cos \phi-X(r, \theta, \xi, \phi)) \\
z_{1} & =\frac{1}{2}(r \cos \theta+Z(r, \theta, \xi)) \\
z_{2} & =\frac{1}{2}(r \cos \theta-Z(r, \theta, \xi))
\end{aligned}
$$

with $X$ and $Z$ as defined in (2.13), (2.15). Then,

$$
\begin{aligned}
\left|\operatorname{det} J T_{2}\right|= & \frac{r \sin \theta}{4} \mid r \sin \theta \sin \phi\left(X_{\xi} Z_{r}-X_{r} Z_{\xi}\right) \\
& +\cos \theta \sin \phi\left(X_{\xi} Z_{\theta}-X_{\theta} Z_{\xi}\right)-\frac{\cos \phi}{\sin \theta} X_{\phi} Z_{\xi} \\
= & \left|y_{1} y_{2}\right| r \sin \theta
\end{aligned}
$$


Now, the transformation $T^{-1}$ is given by the composition of $T_{1}$ and $T_{2}$, from which we conclude that

$$
\left|\operatorname{det} J T^{-1}\right|=r \sin \theta
$$

Hence, we proved the following simple expression for the gain term.

\section{Proposition 2.4}

The operator $Q_{+}(f)$ for Maxwellian molecules can be written in the form

$$
Q_{+}(f)=2^{3} \int_{\mathbb{R}_{+}} \int_{[0,2]} \int_{S^{2}} \int_{S_{x}^{1}} z^{2} f(v-z r n) f(v-z a D(n) u) \mathrm{d} \xi \mathrm{d} \phi \sin \theta \mathrm{d} \theta r \mathrm{~d} r \mathrm{~d} z,
$$

where $n=(\sin \theta \cos \phi, \sin \theta \sin \phi, \cos \theta)$.

Proposition 2.4 is used in the following to derive an $L^{\infty}$-estimate on $Q^{+}(f)$ for $f \in$ $L^{1}\left(I R^{3}\right) \cap L^{\infty}\left(I R^{3}\right)$.

The proof turns out to be quite technical. Hence, we outline shortly the idea how to obtain the estimate: suppose that $f \in L^{1}\left(\mathbb{R}^{3}\right) \cap L^{\infty}\left(\mathbb{R}^{3}\right)$, then one of the terms in the integral on the right hand side of (2.16) can be estimated by the $L^{\infty}$-norm of $\mathrm{f}$.

If we estimate $f(v-z a D(n) u)$ by the $L^{\infty}{ }^{-}$norm we obtain

$$
\left\|Q^{+}(f)\right\|_{\infty} \leq C\|f\|_{\infty}\|f\|_{1} \int_{[0,2]} \frac{1}{r^{2}} \mathrm{~d} r
$$

but the last integral on the right hand side is divergent. Hence, a truncation of the collision kernel becomes necessary to exclude the singularity at $r=0$. A similar idea was already used in [3]. The idea to obtain an estimate without any truncation of the collision kernel is to divide the $r$-intervall in (2.17) into two subdomains: for $r \in[\varepsilon, 2]$ one estimates as given above the term $f(v-z a D(n) u)$ by the $L^{\infty}$-norm, for $r \in[0, \varepsilon]$ the term $f(v-z r n)$ is substituted by the $L^{\infty}$-norm and the remaining integral is bounded by the $L^{1}$-norm of $f$. The main result is formulated in the following theorem.

\section{Theorem 2.5}

Let $f \in L^{1}\left(\mathbb{R}^{3}\right) \cap L^{\infty}\left(\mathbb{R}^{3}\right)$, then

$$
\left\|Q_{+}(f)\right\|_{\infty} \leq 2^{4} \pi\|f\|_{1}\|f\|_{\infty}
$$

\section{Proof}

By Proposition 2.4 we express the gain term $Q_{+}$in the form

$$
Q_{+}(f)=2^{3} \int_{\mathbb{R}_{+}} \int_{[0,2]} \int_{S^{2}} \int_{S_{x}^{1}} z^{2} f(v-z r n) f(v-z a D(n) u) \mathrm{d} \xi \mathrm{d} \phi \sin \theta \mathrm{d} \theta r \mathrm{~d} r \mathrm{~d} z
$$


In order to find the required estimate on $Q_{+}$, we divide the $r$-interval into two subregions and estimate in (2.18) the term $f(v-z r n)$ by the $L^{\infty}$-norm for $r \in[0, \varepsilon]$ and the corresponding term $f(v-z a D(n) u)$ for $r \in[\varepsilon, 2]$. Hence, for $0<\varepsilon<2$, we get

$$
\begin{aligned}
Q_{+}(f) & \leq 2^{4} \pi\|f\|_{\infty} \int_{\boldsymbol{R}_{+}} \int_{[\varepsilon, 2]} \int_{S^{2}} z^{2} f(v-z r n) \mathrm{d} \phi \sin \theta \mathrm{d} \theta r \mathrm{~d} r \mathrm{~d} z \\
& +2^{3}\|f\|_{\infty} \int_{\boldsymbol{R}_{+}} \iint_{[0, \varepsilon]} \int_{S^{2}} z_{S_{x}^{1}} z^{2} f(v-z a D(n) u) \mathrm{d} \xi \mathrm{d} \phi \sin \theta \mathrm{d} \theta r \mathrm{~d} r \mathrm{~d} z
\end{aligned}
$$

Using the substitution $t=z r$, the first integral term on the right hand side can be written as

$$
\iint_{\boldsymbol{R}_{+}} \int_{[\varepsilon, 2]} z_{S^{2}} z^{2} f(v-z r n) \mathrm{d} \phi \sin \theta \mathrm{d} \theta r \mathrm{~d} r \mathrm{~d} z=\left(\frac{1}{\varepsilon}-\frac{1}{2}\right)\|f\|_{1}
$$

For the second integral term in (2.19), we first consider the transformation

$$
\begin{aligned}
& t_{1}=\sin \theta \cos \xi \\
& \xi_{1}=\arctan \left(\frac{\sin \xi}{\cos \theta \cos \xi}\right)
\end{aligned}
$$

which yields

$$
\begin{array}{rl}
\iint_{S^{2}} & f(v-z a D(n) u) \mathrm{d} \xi \mathrm{d} \phi \sin \theta \mathrm{d} \theta= \\
& \int_{[-1,1]} \int_{[0,2 \pi]} \int_{[0,2 \pi]} f\left(v-z a u_{1}\right) \frac{t_{1}}{\cos ^{2} \xi_{1}+t_{1}^{2} \sin ^{2} \xi_{1}} \mathrm{~d} \xi_{1} \mathrm{~d} \phi \mathrm{d} t_{1},
\end{array}
$$

and

$$
u_{1}=\left(\left(1-t_{1}^{2}\right)^{1 / 2} \cos \left(\xi_{1}+\phi\right),\left(1-t_{1}^{2}\right)^{1 / 2} \sin \left(\xi_{1}+\phi\right), t_{1}\right)
$$

Now we substitute $\phi^{\prime}=\xi_{1}+\phi, \xi_{1}^{\prime}=\xi_{1}-\phi$, which yields

$$
\begin{array}{r}
\iint_{[-1,1][0,2 \pi]} \int_{[0,2 \pi]} f\left(v-z a u_{1}\right) \frac{\left|t_{1}\right|}{\cos ^{2} \xi_{1}+t_{1}^{2} \sin ^{2} \xi_{1}} \mathrm{~d} \xi_{1} \mathrm{~d} \phi \mathrm{d} t_{1}= \\
\frac{1}{2} \int_{[-1,1]} \int_{[0,4 \pi]} f\left(v-z a u_{1}^{\prime}\right) A\left(t_{1}, \phi^{\prime}, \xi_{1}^{\prime}\right) \mathrm{d} \phi^{\prime} \mathrm{d} t_{1}
\end{array}
$$

with

$$
u_{1}^{\prime}=\left(\left(1-t_{1}^{2}\right)^{1 / 2} \cos \phi^{\prime},\left(1-t_{1}^{2}\right)^{1 / 2} \sin \phi^{\prime}, t_{1}\right)
$$


and

$$
\begin{aligned}
A\left(t_{1}, \phi^{\prime}, \xi_{1}^{\prime}\right) & =\mathcal{X}\left(\phi^{\prime} \in[0,2 \pi)\right)\left(\int_{\left[-\phi^{\prime}, \phi\right]} \frac{\left|t_{1}\right|}{\cos ^{2}\left(\frac{\phi^{\prime}+\xi_{1}^{\prime}}{2}\right)+t_{1}^{2} \sin ^{2}\left(\frac{\phi^{\prime}+\xi_{1}^{\prime}}{2}\right)} \mathrm{d} \xi_{1}^{\prime}\right) \\
& +\mathcal{X}\left(\phi^{\prime} \in[2 \pi, 4 \pi)\right)\left(\int_{[-4 \pi+\phi, 4 \pi-\phi]} \frac{\left|t_{1}\right|}{\cos ^{2}\left(\frac{\phi^{\prime}+\xi_{1}^{\prime}}{2}\right)+t_{1}^{2} \sin ^{2}\left(\frac{\phi^{\prime}+\xi_{1}^{\prime}}{2}\right)} \mathrm{d} \xi_{1}^{\prime}\right)
\end{aligned}
$$

Applying the relations

$$
\begin{aligned}
& \cos ^{2} x=\frac{1}{2}(1+\cos 2 x) \\
& \sin ^{2} x=\frac{1}{2}(1-\cos 2 x)
\end{aligned}
$$

the $\xi_{1}^{\prime}$-integrals in (2.21) can be estimated by

$$
\begin{array}{r}
\int_{\left[-\phi^{\prime}, \phi^{\prime}\right]} \frac{2\left|t_{1}\right|}{1+t_{1}^{2}+\left(1-t_{1}^{2}\right) \cos \left(\xi_{1}^{\prime}+\phi^{\prime}\right)} \mathrm{d} \xi_{1}^{\prime} \leq 4 \pi \\
\int_{\left[-4 \pi+\phi^{\prime}, 4 \pi-\phi^{\prime}\right]} \frac{2\left|t_{1}\right|}{1+t_{1}^{2}+\left(1-t_{1}^{2}\right) \cos \left(\xi_{1}^{\prime}+\phi^{\prime}\right)} \mathrm{d} \xi_{1}^{\prime} \leq 4 \pi
\end{array}
$$

and, using $t=z a$, it remains to compute

$$
\begin{aligned}
& \int_{\mathbb{R}_{+}[0, \varepsilon]} \int_{S^{2}} z^{2} f\left(v-z a u_{1}^{\prime}\right) r \mathrm{~d} \phi^{\prime} \mathrm{d} t_{1} \mathrm{~d} r \mathrm{~d} z \\
& =\int_{\boldsymbol{R}_{+}} \int_{S^{2}} t^{2} f\left(v-t u_{1}^{\prime}\right)\left(\int_{0, \varepsilon]} \frac{r}{8\left(1-\frac{r^{2}}{4}\right)^{3 / 2}} \mathrm{~d} r\right) \mathrm{d} \phi^{\prime} \mathrm{d} t_{1} \mathrm{~d} t \\
& =\left(\frac{1}{\sqrt{4-\varepsilon^{2}}}-\frac{1}{2}\right) \int_{\boldsymbol{R}_{+}} \int_{S^{2}} f\left(v-t u_{1}^{\prime}\right) t^{2} \mathrm{~d} \phi^{\prime} \mathrm{d} t_{1} \mathrm{~d} t \\
& =\left(\frac{1}{\sqrt{4-\varepsilon^{2}}}-\frac{1}{2}\right)\|f\|_{1}
\end{aligned}
$$

Hence, we can summarize the estimates to get

$$
Q_{+}(f) \leq C(\varepsilon)\|f\|_{\infty}\|f\|_{1}
$$

where

$$
C(\varepsilon)=2^{4}\left(\frac{1}{\varepsilon}+\frac{4}{\sqrt{4-\varepsilon^{2}}}-\frac{5}{2}\right) \pi
$$


The same technique as described above may be used to derive an estimate on a more general gain term involving two density functions $f$ and $g$. We define for $f, g \in L^{1}\left(I R^{3}\right) \cap$ $L^{\infty}\left(I R^{3}\right)$ the operator $Q_{+}(f, g)$ by

$$
Q_{+}(f, g)=2 \int_{\mathbb{R}^{3}} \int_{[0, \pi / 2]} \int_{[0,2 \pi]}\left(f\left(v^{\prime}\right) g\left(v_{*}^{\prime}\right)+g\left(v^{\prime}\right) f\left(v_{*}^{\prime}\right)\right) \cos \theta_{1} \mathrm{~d} \phi_{1} \sin \theta_{1} \mathrm{~d} \theta_{1} \mathrm{~d} v_{*}
$$

Then, an immediate estimate on $Q_{+}(f, g)$ is given by the following theorem.

\section{Theorem 2.6}

Let $f, g \in L^{1}\left(\mathbb{R}^{3}\right) \cap L^{\infty}\left(\mathbb{R}^{3}\right)$, then

$$
\left\|Q_{+}(f, g)\right\|_{\infty} \leq 2^{3} \pi\left(\|f\|_{1}\|g\|_{\infty}+\|g\|_{1}\|f\|_{\infty}\right)
$$

Proof

In the center of mass system, using Proposition $2.4, Q_{+}(f, g)$ can be written in the form

$$
\begin{aligned}
Q_{+}(f, g)= & 2^{2} \iint_{\mathbb{R}_{+}} \iint_{[0,2]} \int_{S^{2}} z_{S_{x}^{1}}(f(v-z r n) g(v-z a D(n) u) \\
& +g(v-z r n) f(v-z a D(n) u)) \mathrm{d} \xi \mathrm{d} \phi \sin \theta \mathrm{d} \theta r \mathrm{~d} r \mathrm{~d} z
\end{aligned}
$$

Following the same steps as in the proof of Theorem 2.5 yields (2.22).

\section{The General Case}

In the following we consider a more general form of expression (2.3) given by

$$
Q_{+}(f)=4 \int_{\mathbb{R}^{3}} \int_{[0, \pi / 2]} \int_{[0,2 \pi]}\left\|v-v_{*}\right\|^{\alpha} f\left(v^{\prime}\right) f\left(v_{*}^{\prime}\right) \cos \theta_{1} \mathrm{~d} \phi_{1} \sin \theta_{1} \mathrm{~d} \theta_{1} \mathrm{~d} v_{*},
$$

with $-1 \leq \alpha \leq 1$, which includes the hard-sphere-interaction potential for $\alpha=1$. Applying the results of Section 2, it is straightforward to derive the following expression for $Q_{+}(f)$.

\section{Proposition 3.1}

The operator $Q_{+}(f)$ in (3.1) can be written in the form

$$
Q_{+}(f)=2^{3+\alpha} \int_{\mathbb{R}_{+}} \int_{[0,2]} \int_{S^{2}} \int_{S_{x}^{1}} z^{2+\alpha} f(v-z r n) f(v-z a D(n) u) \mathrm{d} \xi \mathrm{d} \phi \sin \theta \mathrm{d} \theta r \mathrm{~d} r \mathrm{~d} z
$$

where $n=(\sin \theta \cos \phi, \sin \theta \sin \phi, \cos \theta)$. 


\section{Proof}

In the center of mass system, the general form given in (3.1) can be written as

$$
Q_{+}(f)=\iint_{\boldsymbol{R}^{3}[0, \pi]} \int_{[0,2 \pi]}\left\|v-v_{*}\right\|^{\alpha} f\left(v^{\prime}\right) f\left(v_{*}^{\prime}\right) \mathrm{d} \phi \sin \theta \mathrm{d} \theta \mathrm{d} v_{*}
$$

and following the same steps as described in Section 2 yields equation (3.2).

\section{Theorem 3.2}

Let $f \in L^{\infty}\left(\mathbb{R}^{3}\right)$, then there exists $C<\infty$, such that

$$
Q_{+}(f)(v) \leq C\|f\|_{\infty} L(f)(v) \quad \forall v \in \mathbb{I}^{3}
$$

\section{Proof}

Using (3.2), the proof follows the same arguments as in Theorem 2.5. Hence, for $v \in \mathbb{R}^{3}$ we have the estimate

$$
\begin{aligned}
Q_{+}(f)(v) & \leq 2^{4+\alpha} \pi\|f\|_{\infty}\left(\int_{[\varepsilon, 2]} \frac{1}{r^{2+\alpha}} \mathrm{d} r\right) \int_{R^{3}}\left\|v-v_{*}\right\|^{\alpha} f\left(v_{*}\right) \mathrm{d} v_{*} \\
& +2^{6+\alpha} \pi\|f\|_{\infty}\left(\int_{[0, \varepsilon]} \frac{r}{a^{3+\alpha}} \mathrm{d} r\right) \int_{\mathbb{R}^{3}}\left\|v-v_{*}\right\|^{\alpha} f\left(v_{*}\right) \mathrm{d} v_{*} \\
& =C(\varepsilon)\|f\|_{\infty} L(f)(v)
\end{aligned}
$$

for $0<\varepsilon<2$ and $a$ as given in (2.10).

\section{Remark 3.3}

It should be noticed, that the result of Theorem 2.5 is included in the theorem above: if $\alpha=0$, one computes that $L(f)(v)=\|f\|_{1}$ for all $v \in \mathbb{R}^{3}$.

\section{Remark 3.4}

For the general case, it is obvious that the gain term $Q_{+}$is unbounded in the $L^{\infty}$-norm: if $\alpha=1$, i.e. the hard-sphere-interaction potential, and $f=f_{m}$ a Maxwellian density function, one computes (see [2])

$$
L\left(f_{m}\right)(v)=\frac{1}{\sqrt{\beta \pi}} \mathrm{e}^{-\beta v^{2}}+\left(\frac{1}{2 \beta}+|v|^{2}\right) \frac{\operatorname{erf}(\sqrt{\beta}|v|)}{|v|}
$$

where

$$
f_{m}(v)=\left(\frac{\beta}{\pi}\right)^{3 / 2} \mathrm{e}^{-\beta v^{2}}, \quad v \in \mathbb{R}^{3}, \beta>0
$$

Hence, $Q_{+}\left(f_{m}\right)=f_{m} L\left(f_{m}\right)$ is unbounded in $L^{\infty}$. 
In analogy to Section 2 , we now consider the gain term $Q_{+}(f, g)$ given by

$$
Q_{+}(f, g)=4 \int_{\mathbb{R}^{3}} \int_{[0, \pi / 2]} \int_{[0,2 \pi]}\left\|v-v_{*}\right\|^{\alpha}\left(f\left(v^{\prime}\right) g\left(v_{*}^{\prime}\right)+g\left(v^{\prime}\right) f\left(v_{*}^{\prime}\right)\right) \cos \theta_{1} \mathrm{~d} \phi_{1} \sin \theta_{1} \mathrm{~d} \theta_{1} \mathrm{~d} v_{*},
$$

Then, the estimate on $Q_{+}(f, g)$ can be written as follows.

\section{Theorem 3.5}

Let $f, g \in L^{1}\left(I R^{3}\right) \cap L^{\infty}\left(I R^{3}\right)$, then

$$
Q_{+}(f, g) \leq C(\varepsilon)\left(\|f\|_{1}\|g\|_{\infty}+\|g\|_{1}\|f\|_{\infty}\right)
$$

\section{Proof}

With Proposition 3.1, the result is an immediate consequence of Theorem 3.2.

\section{References}

[1] C. Cercignani, R. Illner and M. Pulvirenti: The Mathematical Theory of Dilute Gases, Springer, New York, 1994.

[2] R. Illner and J. Struckmeier, Boundary Value Problems for the Steady Boltzmann Equation, preprint, to appear in J. of Stat. Physics (1996).

[3] R.J. DiPerna and P.L. Lions: On the Cauchy problem for the Boltzmann equation: Global existence and weak stability, Annals of Mathematics (1989). 